\title{
Morphological Location of Mandibular and Mental Foramen in Teenagers: A Cone-beam Computed Tomography Study
}

\author{
Nimisha Vathariparambath ${ }^{1}$, Navin H Krishnamurthy ${ }^{2}$, Nagarathna Chikkanarasaiah ${ }^{3}$
}

\begin{abstract}
Introduction: Pain control is an important aspect of the behavior management of pediatric dental patients. Local anesthesia is still a widely used technique for pain control in pediatric dental treatment in which inferior alveolar nerve block is fundamental for achieving local anesthesia for mandibular restorative and surgical procedures. The assessment of the mandibular foramen (MF) and mental foramen (MeF) is of considerable importance for mandibular nerve anesthesia. There may be failure of mandibular nerve block due to improper assessment of the location of the MF and MeF resulting in painful treatment procedures especially in children. A considerable variation in the location of the MF and MeF was reported among different population, in different ages, and even within the same individual on two sides.

Aim and objective: To determine the location of the MF and MeF in teenagers using cone-beam computed tomography (CBCT).

Materials and methods: A total of $100 \mathrm{MF}$ and MeF (right and left) were obtained from $100 \mathrm{CBCT}$ images of children (13-18 years). Distance from MF to the anterior border (AB) of ramus $(A)$, posterior border of ramus $(P)$ and inferior border of the mandible (MI), most superior point of the curvature of mandibular notch $(\mathrm{MN})$, occlusal plane of the mandibular permanent molars $(\mathrm{O})$, and the distance from MeF to lower border of mandible (BM) and to the alveolar crest (AC) were measured.

Results: There was a significant increase in A-MF, MI-MF, MN-MF, and O-MF values with age. A-MF and MI-MF values of females were statistically higher than those of males and MF was located at the occlusal plane at 13 years and then moves upward. There was a significant difference in BM-MeF value with age $(p=0.05)$.

Conclusion: The location of the MF is just posterior to the middle of the ramus and MF and MeF are shifting posterosuperiorly with increasing age. Keywords: Cone-beam computed tomography, Mandibular foramen, Mental foramen.

Journal of Health Sciences \& Research (2020): 10.5005/jp-journals-10042-1088
\end{abstract}

\section{INTRODUCTION}

The assessment of the mandibular foramen (MF) and mental foramen (MeF) is of considerable importance for mandibular nerve anesthesia. The improper evaluation of the location of the MF and MeF may result in the failure of mandibular nerve block leading to painful treatment procedures especially in children. The location of the MF and MeF shows considerable variation among different population, in different age, sex, and even within the same individual on two sides.

The comfort of many dental treatments in children especially depends on achieving excellent local anesthesia. ${ }^{1}$ Anesthesia in the mandible may be associated with some difficulty and success of this technique depends on the proximity between the anesthetic needle and the mandibular anatomical landmarks. ${ }^{2}$ The failure in anesthesia of inferior alveolar nerve block lies in the inappropriate setting of the needle, due to the inaccurate location of the anatomic structures like MF and MeF. Similarly, the accurate identification of $\mathrm{MeF}$ is important for all the clinical procedure in the anterior region of the mandible. ${ }^{2}$

There are no anatomical landmarks for locating the MeF and the foramen cannot be clinically visualized or palpated, as a result $\mathrm{MeF}$ is difficult to locate and the reported anatomical position of the MeF has been variable. ${ }^{3,4}$ Clinically, the mental bundle could be injured during surgical procedures, resulting in paresthesia or anesthesia. ${ }^{3}$ Most studies and textbook, however, describe the location of the MeF as being below the apex of the second premolar or between the apices of the first and second premolars. ${ }^{3,5}$ The
${ }^{1-3}$ Department of Pediatric and Preventive Dentistry, RajaRajeswari Dental College and Hospital, Bengaluru, Karnataka, India

Corresponding Author: Nimisha Vathariparambath, Department of Pediatric and Preventive Dentistry, RajaRajeswari Dental College and Hospital, Bengaluru, Karnataka, India, e-mail: nimmi.nimm@gmail.com How to cite this article: Nimisha VP, Krishnamurthy $\mathrm{NH}$, Chikkanarasaiah N. Morphological Location of Mandibular and Mental Foramen in Teenagers: A Cone-beam Computed Tomography Study. J Health Sci Res 2020;11(1):1-5.

Source of support: Nil

Conflict of interest: None

radiographic location of the MF and MeF may not be the exact since radiographs give a two-dimensional (2D) view of three-dimensional (3D) objects. ${ }^{4}$

Using conventional radiographic techniques, the appearance of the MeF may result in a misdiagnosis of a radiolucent lesion in the apical area of mandibular premolar teeth. Cone-beam computed tomography (CBCT) has probably been one of the most revolutionary innovations in the field of pediatric dentistry, and it provides a novel platform for imaging of maxillofacial area. It provided an imaging solution that has none of the projection errors associated with magnification and none of the superimposition problems associated with traditional panoramic imaging. It is particularly suitable in the evaluation of jaws, as 3D 
visualization and high-resolution analysis of the entire mandible provide adequate information to localize anatomic structures like MF and MeF. ${ }^{6}$ The relative position of the inferior alveolar canal and its mental and mandibular foramina varied with age and show sexual dimorphism, which were indicated in previous studies. Studies undertaken on the morphological location of the MF and MeF using CBCT in Indian pediatric population are very few or scarce. ${ }^{1}$

Hence, the aim of this study was to evaluate the morphological location of the MF and MeF in mixed dentition period using CBCT.

\section{Materials and Methods}

This retrospective study was performed using 50 CBCT images of children in the age group of 6-14 years, obtained from the institutional database of Rajarajeswari Dental College and Hospital, Bengaluru. A total of $100 \mathrm{MF}$ and MeF, both right and left side, were analyzed on various parameters. The subject was divided into two groups-Group I: Age 13-15 years and group II: 16-18 years. The CBCT images were reconstructed with the help of ONDEMAND 3D and Scanora software (CBCT MACHINE: SCANORA 3D, SOREDEX, Finland) at $90 \mathrm{kVp}$ with an exposure time of 8 seconds. The images were then evaluated to determine the location of the MF and MeF. Fifty CBCT images of mandible that was taken for valid diagnostic reasons or treatment were collected and analyzed. In order to ensure the integrity, a good quality of the CBCT mandibular images with the following criteria was selected. Cone-beam computed tomography images of age group 13-18 years, premolar and molar regions of both side of the mandible, no periapical pathology of teeth, developmental or congenital anomalies in soft or hard tissues in the mandible, and presence of all mandibular teeth.

Cone-beam computed tomography images with pathologies, such as, cyst, tumors, bone loss in the mandibular premolar and molar regions. Evidence of fracture or history of trauma to the mandibular premolar and molar regions. Showing errors and artifacts obscuring visibility of structures in the mandible were excluded from the study.

\section{INTERPRETATION OF IMAGES}

All the images were assessed and measured using ONDEMAN 3D and SCANORA SOFTWARE of the CBCT machine. The panoramic and coronal images were used for locating the MF and MeF, respectively. The following measurements were made using the measuring tool on ONDEMAND 3D software. A panoramic view was reconstructed along the inferior alveolar canal. The location of the MF was investigated and the following measurements were recorded (Fig. 1).

- The shortest distance between the anterior border (A) and MF.

- The shortest distance between the posterior border (P) and MF.

- The shortest distance between the inferior border of mandible (MI) to MF.

- The shortest distance between the most superior point of the curvature of the mandibular notch (MN) and MF.

- The distance between the straight line of the cusps of the mandibular permanent molars and MF (O).

A coronal view was reconstructed. The location of the MeF was investigated and the following measurements were recorded (Fig. 2).

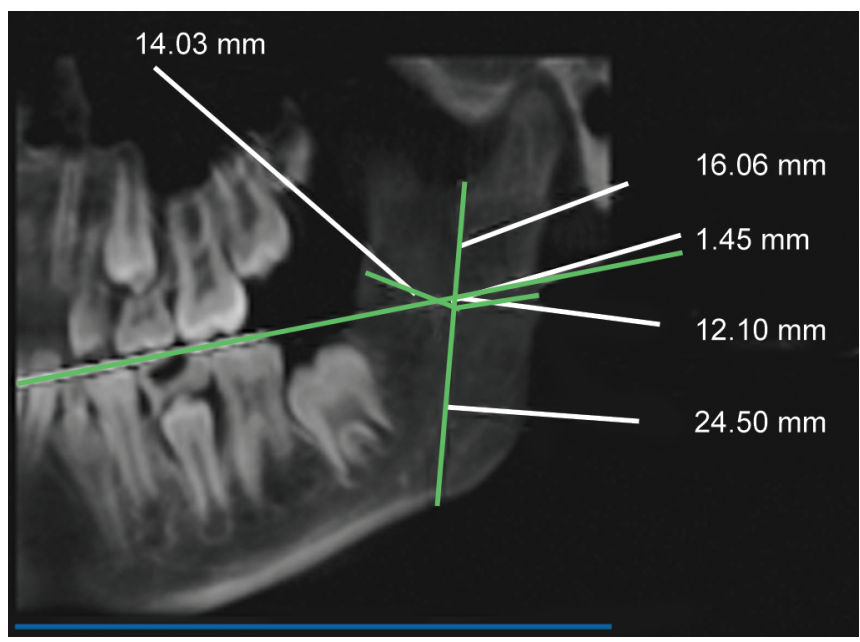

Fig. 1: Location of the mandibular foramen. Landmarks used in the present study. Anterior border of the ramus $(A)$, posterior border of the ramus $(\mathrm{P})$, inferior border of mandible $(\mathrm{MI})$, the most superior point of the curvature of the mandibular notch (MN), straight line of the cusps of the mandibular permanent molars, and mandibular foramen $(\mathrm{O})$

Three tangents were drawn, one at the superior margin of the MeF other at the crest of the alveolar bone and one at the base of the mandible. The distance between these three lines was recorded, which indicates the distance of the foramen from the crestal bone and the base of the mandible.

The differences, if any in morphometry of these structures with regard to age and sex were noted. All measurements were recorded in millimeters. Student paired $t$ test was used to compare the rightand left-sided linear distances related to MF in different age groups.

One-way analysis of variance (ANOVA) test was used to compare the various linear distances related to MF based on different age groups and Mann-Whitney $U$ test was used. The level of significance was set at $p<0.05$. The study data were analyzed using SPSS (Statistical Package for Social Sciences) v.22 (IBM, Corp.,) for Windows.

\section{Results}

There was a significant increase in A-MF, MI-MF, MN-MF, and O-MF values with age. A-MF and MI-MF values of females were statistically higher than those of males and MF was located at the occlusal plane at 13 years and then moves upward. There was a significant difference in BM-MeF value with age $(p=0.05)$ and there is no significant difference based on gender and side (Fig. 3).

\section{Discussion}

The determination of the accurate anatomic location of the MF and MeF is essential to avoid any untoward outcomes, such as, hemorrhage and permanent neurological damage caused by the transection of the neurovascular bundle during the administration of local anesthesia or surgical procedures in the mandible. ${ }^{7}$ Its accurate position enables more effective local anesthesia and improved child cooperation.

The location of the MF and MeF could change during the growth and development of the jaw especially during mixed dentition period. ${ }^{1,8}$ The importance of the MF and MeF in the success of mandibular nerve block was revealed in previous studies, but 


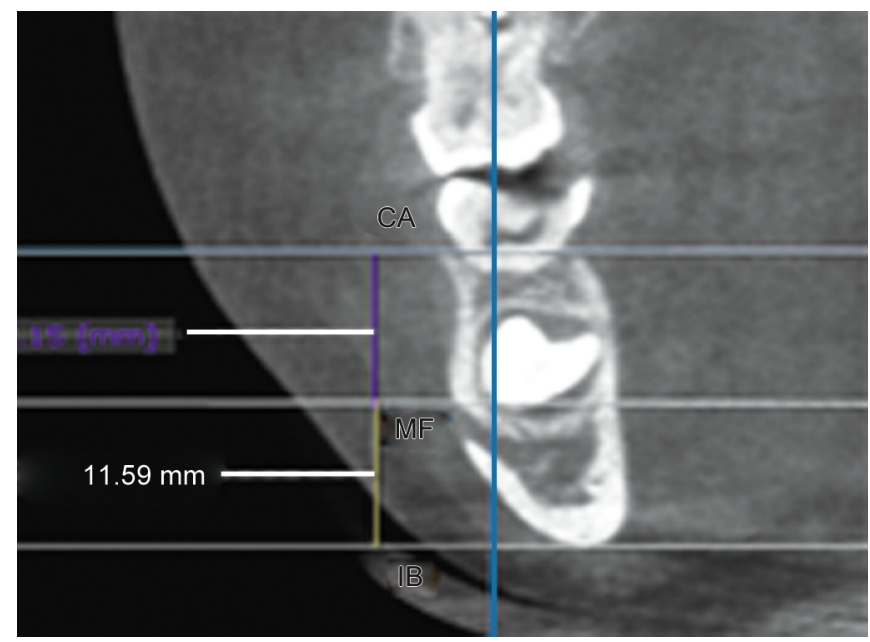

Fig. 2: Location of the mental foramen. Landmarks used in the present study. The superior margin of the mental foramen (MeF), the crest of the alveolar bone $(\mathrm{AC})$, and the base of the mandible (MB)

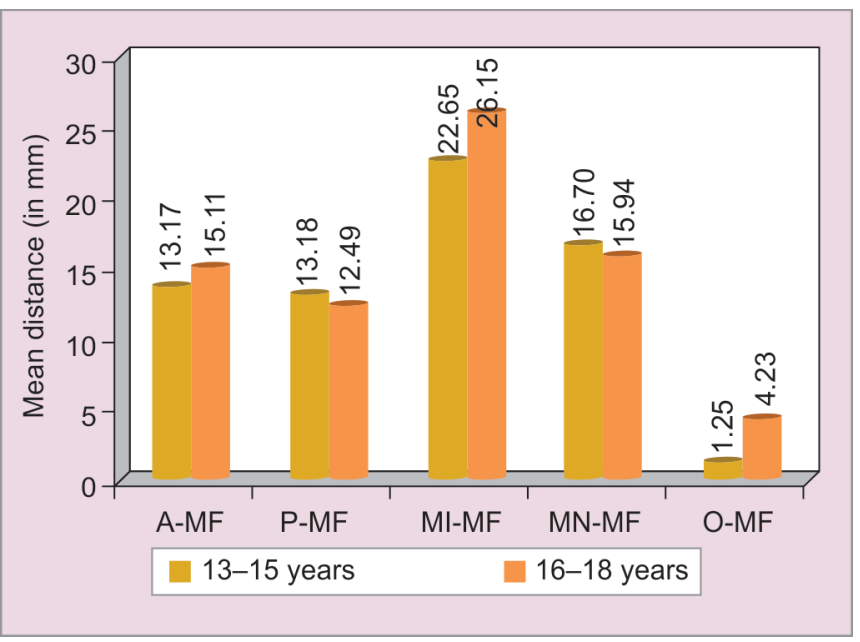

Fig. 4: Age-wise comparison of different anatomical location of the mandibular foramen among study subjects

studies undertaken on the morphological location of the MF and $\mathrm{MeF}$ in Indian pediatric population are very few or scarce., ${ }^{1,2}$

Studies for determining the localization of the MF and MeF have commonly been carried out using conventional radiographic technique like panoramic radiographs; however, they have some disadvantages, such as, loss of definition, image magnification, and less accurate diagnostic value. ${ }^{9}$

Cone-beam computed tomography has been considered a "Gold Standard" as it provides clear and accurate images of structures, and therefore is extremely useful for assessing the bone component. ${ }^{6,10}$ Thus, the aim of the present study was to determine the location of the MF and MeF in mixed dentition period using $C B C T$.

The knowledge about the location of the MF from the anterior border $(A B)$ and from occlusal plane helps the dentist to locate the position of inferior alveolar nerve entry into the foramen accurately. Similarly, the knowledge of localization of the MF from the posterior border of the ramus and the inferior border of the mandible and condyle would be helpful in the advanced extraoral

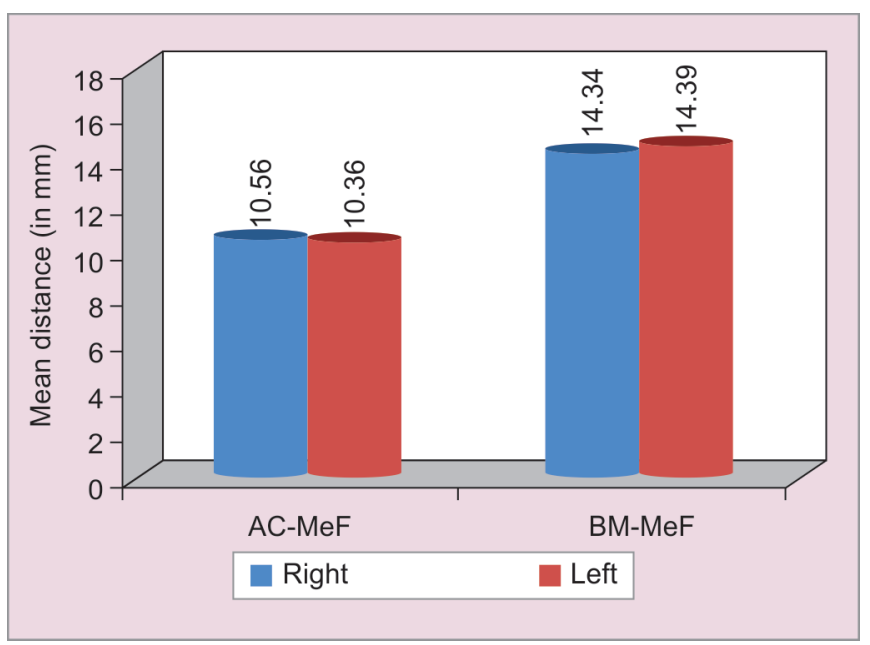

Fig. 3: Comparison of mean values of different anatomical locations of mental foramen between right and left sides using student paired $t$ test

inferior alveolar nerve block technique. ${ }^{2}$ Lindh et al. measured the distances between the superior border of the canal and the alveolar crest and between the mandibular base and the inferior border of the canal, and in addition the height of the canal in his study and concluded that tomography gave more accurate values than panoramic techniques. ${ }^{7}$ Study conducted by Thangavelu et al. ${ }^{2}$ reported that the MF is at a distance of $19 \mathrm{~mm}$ (SD $2.34 \mathrm{~mm}$ ) from AB. In our study, the MF was found to be at a mean distance of 13.02 $\mathrm{mm}$ (SD $1.62 \mathrm{~mm}$ ) at the age of 13-15 years and $13.91 \mathrm{~mm}$ (SD 2.21 $\mathrm{mm}$ ) from $A B$ of ramus at the age of 15-18 years; with significant difference between male and female and without significant difference between the right and left side (Fig. 4).

In the present study, the MF was located posterior to the middle of the ramus and superiorly from the midpoint of vertical height (MN-MI) (Table 1). Whereas in other studies, it was found that the MF is located just posterior to the middle of the ramus. ${ }^{1,11}$ Hayward et al. reported that the MF was located in the third quadrant of the ramus. ${ }^{12}$ Thangavelu et al. reported that the MF is $3 \mathrm{~mm}$ superiorly from the midpoint of vertical height. ${ }^{2}$ The distance of P-MF value was reported to be approximately 13.18 and $12.49 \mathrm{~mm}$ in the age group of 13-15 and 16-18 years, respectively. The distance of MN-MF value was reported to be approximately 22.65 and $26.15 \mathrm{~mm}$ in the age group of 13-15 and 16-18 years, respectively, and there is a statistically difference between genders (Fig. 4 and Tables 1 and 2). Krishnamurthy et al. ${ }^{13}$ reported that the MF is $13.0-13.9 \mathrm{~mm}$ from deepest point of coronoid notch for 13-15 years of age group, which supports our study. Altunsoy et al. examined children and youth ranging from 8 to 18 years and study reported that the P-MF and MN-MF values were statistically increasing with age. ${ }^{1}$ Afsar et al. reported that no significant difference between male and female values for the distance measured from the MF to the landmarks. ${ }^{11}$ Altunsoy et al. found that there is a statistically increase in MI-MF value with age and significant difference between genders, but present study shows no significant difference in this value but shows sex difference. ${ }^{1}$

The occlusal plane is an important anatomical landmark for the identification of MF. The location of the MF is shifting superiorly from the occlusal plane and the O-MF value shows a statistical difference with age. Hwang et al. reported that the MF was located $4.12 \mathrm{~mm}$ below the occlusal plane at the age of 3 years and subsequently 
Localization of MF and MeF in Indian Pediatric Population

Table 1: Gender-wise comparison of different anatomical locations of mandibular foramen among study subjects using independent student $t$ test, and genderwise comparison of mean distance of mandibular foramen (in $\mathrm{mm}$ ) from point $\mathrm{O}$ using Mann-Whitney $U$ test

\begin{tabular}{|c|c|c|c|c|c|c|c|}
\hline \multicolumn{8}{|c|}{ Gender-wise comparison of mean distance of mandibular foramen (in $\mathrm{mm}$ ) from different anatomical points using student paired $\mathrm{t}$ test } \\
\hline Points & Gender & $N$ & Mean & $S D$ & Mean diff & $t$ & p value \\
\hline \multirow[t]{2}{*}{ A-MF } & Males & 26 & 14.83 & 1.64 & 1.09 & 2.308 & $0.03^{*}$ \\
\hline & Females & 24 & 13.74 & 1.70 & & & \\
\hline \multirow[t]{2}{*}{ P-MF } & Males & 26 & 12.78 & 1.45 & -0.27 & -0.597 & 0.55 \\
\hline & Females & 24 & 13.04 & 1.74 & & & \\
\hline \multirow[t]{2}{*}{ MI-MF } & Males & 26 & 24.72 & 2.63 & 1.40 & 1.953 & $0.04^{*}$ \\
\hline & Females & 24 & 23.32 & 2.44 & & & \\
\hline \multirow[t]{2}{*}{ MN-MF } & Males & 26 & 16.52 & 1.15 & 0.26 & 0.669 & 0.51 \\
\hline & Females & 24 & 16.26 & 1.59 & & & \\
\hline \multicolumn{8}{|c|}{ Gender-wise comparison of mean distance of mandibular foramen (in $\mathrm{mm}$ ) from point $\mathrm{O}$ using Mann-Whitney $U$ test } \\
\hline Points & Gender & $N$ & Mean & $S D$ & Mean diff & $Z$ & p value \\
\hline \multirow[t]{2}{*}{ O-MF } & Males & 26 & 2.56 & 2.12 & 0.23 & -0.495 & 0.65 \\
\hline & Females & 24 & 2.32 & 1.91 & & & \\
\hline
\end{tabular}

${ }^{*} p<0.05$

Table 2: Comparison of mean values of different anatomical locations of mandibular foramen between right and left sides using student paired $t$ test and comparison of mean distance of mandibular foramen (in $\mathrm{mm}$ ) from point $\mathrm{O}$ between right and left sides using Wilcoxon signed-rank test

Comparison of mean distance of mandibular foramen (in $\mathrm{mm}$ ) from different anatomical points between right and left sides using student paired $t$ test

\begin{tabular}{|c|c|c|c|c|c|c|c|}
\hline Regions & Sides & $N$ & Mean & $S D$ & Mean diff & $t$ & $p$ value \\
\hline \multirow[t]{2}{*}{ A-MF } & Right & 50 & 14.35 & 1.91 & 0.07 & 0.614 & 0.54 \\
\hline & Left & 50 & 14.27 & 1.66 & & & \\
\hline \multirow[t]{2}{*}{ P-MF } & Right & 50 & 13.04 & 1.69 & 0.27 & 1.920 & 0.06 \\
\hline & Left & 50 & 12.77 & 1.62 & & & \\
\hline \multirow[t]{2}{*}{ MI-MF } & Right & 50 & 24.08 & 2.67 & 0.06 & 0.688 & 0.50 \\
\hline & Left & 50 & 24.02 & 2.59 & & & \\
\hline \multirow[t]{2}{*}{ MN-MF } & Right & 50 & 16.41 & 1.35 & 0.02 & 0.215 & 0.83 \\
\hline & Left & 50 & 16.38 & 1.47 & & & \\
\hline
\end{tabular}

Comparison of mean distance of mandibular foramen (in $\mathrm{mm}$ ) from point O between right and left sides using Wilcoxon signed-rank test

\begin{tabular}{llllllll}
\hline Regions & Sides & $N$ & Mean & SD & Mean diff & $Z$ & $p$ value \\
\hline O-MF & Right & 50 & 2.43 & 2.03 & -0.03 & -0.717 & 0.47 \\
& Left & 50 & 2.46 & 2.00 & & & \\
\hline
\end{tabular}

moved upward with age. It had reached approximately the same level as the occlusal plane by 9 years and the foramen continued to move upward to $4.16 \mathrm{~mm}$ above the occlusal plane in the adult patients. ${ }^{14}$ According to other study, MF moves superiorly above the occlusal plane by 13 years of age. ${ }^{15} \mathrm{~A}$ study conducted by Pereira et al. by examining 4- to 12-year-old children, it was found that in $65 \%$ of cases MF was detected below the occlusal plane. ${ }^{16}$ The present study showed a statistically significant difference in the O-MF value with age and is at the occlusal plane at 13 years of age. According to Altunsoy et al., it was found that the location of the MF is $2.5-3.6 \mathrm{~mm}$ above the occlusal plane of the molars and there were no statistically changes in the location of the MF with age. ${ }^{1}$ The significant difference in BM-MeF value with age was noted in our study. Location of the MeF was bilaterally symmetric (Fig. 3) and no gender difference was found. The position of the MeF was shifting superiorly with increasing age. There was decrease in AC-MeF value, from $10.60 \mathrm{~mm}$ at the age of $13-15$ years to 10.25 $\mathrm{mm}$ at $16-18$ years. There is a significant increase in $\mathrm{BM}-\mathrm{MeF}$ value from $13.58 \mathrm{~mm}$ in group 1 to $15.54 \mathrm{~mm}$ in the group II (Table 3).
Mental foramen opens midway between upper and lower border at the age of 13 years because the alveolar and subalveolar parts are equally developed. And in most of the cases, it was located between the first and second primary molars. Gershenson et al. reported that majority of the MeF was located opposite the first deciduous molar root. ${ }^{17}$ From our study, we found that, with increasing age, the vertical measurement of the sagittal size of the structures of the mandible increase; therefore, the distance from the MF and MeF to the landmarks can vary, as shown in this study and previous studies. Thus, it is difficult to say that there is a specific value for any distance or ratio of the MF and this value can vary in different populations. These variances may be based on differences in population and/or measurement techniques. Based on the obtained results, the location of the MF is just posterior to the middle of the ramus; it reaches the occlusal plane of the molars at 13 years of age and it moves posterosuperior and the A-MF, P-MF, MN-MF, MI-MF, and O-MF values increase with age. Similarly, the location of the MeF moves posterosuperior with increasing age and MF and MeF maintain bilateral symmetry. 
Localization of MF and MeF in Indian Pediatric Population

Table 3: Age-wise comparison of different anatomical locations of mental foramen among study subjects using independent student $t$ test

\begin{tabular}{llllllll}
\hline Points & Age group & $N$ & Mean & SD & Mean diff & $t$ & 1.077 \\
\hline AC-MeF & $13-15$ years & 30 & 10.60 & 1.21 & 0.35 & 0.29 & \\
& $16-18$ years & 20 & 10.25 & 0.98 & & -5.049 & $<0.001^{*}$ \\
BM-MeF & $13-15$ years & 30 & 13.58 & 1.12 & -1.96 & & \\
& $16-18$ years & 20 & 15.54 & 1.64 & & & \\
\hline
\end{tabular}

${ }^{*} p<0.05$

\section{Conclusion}

Based on the values obtained, it can be concluded that the location of the MF and MeF varied according to age and gender, which was also determined by size, width, height, growth, and development of each child in various age groups. The location of the MF is just posterior to the middle of the ramus and MF and MeF are shifting superiorly with increasing age and it reaches occlusal plane by 13 years of age. So, a thorough knowledge of the anatomical structures is of at most important for successful treatment and prognosis especially in children.

\section{LiMITATION}

The limitation of our study would be based on the fact that the radiographs were obtained from the database; hence, the nutritional status and the general health of each subject could not be assessed. Nutritional status and general health of the child do play an influence on his or her growth and development and the sample size was less. This study also missed out to assess the location of the MF on the ethnic grounds other than the South Indian population.

\section{Why is this StUdy Important?}

The position of the MF and MeF is very important while performing local anesthesia of mandible and its position changes according to age especially during growth spurts. Failure to achieve proper nerve block leading to repeated injection of the local anesthetic solution will not only pose a behavior problem in children but can also lead to systemic toxic level of anesthetic solution being administered.

When a dentist is aware of the location of the MF and MeF based on age and gender of the patient may help to promote successful and pain-free treatment which also reduced the risk of complications. In the future, more research conducted on the localization of the MF and MeF from the different anatomical location using CBCT and wide patient groups is needed.

\section{Acknowledgments}

We would like to thank Dr Balaji P, Head of the Department of Oral Medicine and Radiology, for granting us access to the database of orthopantomographs, for successful conductance of the study. We also extend our gratitude to the management people of RajaRajeswari Dental College, Bengaluru, Karnataka, India, for their constant encouragement and support.

\section{References}

1. Altunsoy M, Aglarci OS, Ok E, et al. Localization of the mandibular foramen of 8-18 years old children and youths with cone-beam computed tomography. J Pediatr Dent 2014;2(2):44-48. DOI: 10.4103/2321-6646.137677.

2. Thangavelu K, Kannan R, Kumar NS, et al. Significance of localization of mandibular foramen in an inferior alveolar nerve block. J Nat Sci Biol Med 2012;3(2):156-160. DOI: 10.4103/0976-9668.101896.

3. Ngeow WC, Yuzawati Y. The location of the mental foramen in a selected Malay population. J Oral Sci 2003;45(3):171-175. DOI: 10.2334/josnusd.45.171.

4. Phillips JL, Weller RN, Kulild JC. The mental foramen: Part 1. Size,orientation and positional relationship to the mandibular second premolar. J Endod 1990;16(5):221-223. DOI: 10.1016/S00992399(06)81674-2.

5. Philips JL, Weller RN, Kulild JC. The mental foramen: Part II. Radiographic position in relation to the mandibular second premolar. J Endod 1992;18(6):271-274. DOI: 10.1016/S0099-2399(06)80953-2.

6. Cantekin K, Sekerci AE, Miloglu O, et al. Identification of the mandibular landmarks in a pediatric population. Med Oral Patol Oral Cir Bucal 2014;19(2):e136-e141. DOI: 10.4317/medoral.18980.

7. Lindh C, Petersson A, Klinge B. Measurements of distances related to the mandibular canal in radiographs. Clin Oral Implants Res 1995;6(2):96-103. DOI: 10.1034/j.1600-0501.1995.060205.x.

8. Gungor K, Ozturk M, Semiz M, et al. A radiographic study of location of mental foramen in a selected Turkish population on panoramic radiograph. Collegium Antropologicum Coll Antropol 2006;30(4):801-805.

9. Ono E, Médice Filho E, de Moraes LC, et al. Anteroposterior location of the mandibular foramen of 7 to 12 year-old children in panoramic radiographs. Brazil Dent Sci 2010;8(2). DOI: 10.14295/bds.2005. v8i2.258.

10. Przesmycka A, Tomczyk J. Differentiation of root canal morphology-a review of the literature. Anthropolog Rev 2016;79(3):221-239. DOI: 10.1515/anre-2016-0018.

11. Afsar A, Haas DA, Rossouw PE, et al. Radiographic localization of mandibular anesthesia landmarks. Oral Surg Oral Med Oral Pathol Oral Radiol Endod 1998;86(2):234-241. DOI: 10.1016/S10792104(98)90131-7.

12. Hayward J, Richardson ER, Malhotra SK. The mandibular foramen: its anteroposterior position. Oral Surg, Oral Med, Oral Pathol Oral Radiol 1977;44(6):837-843.

13. Krishnamurthy $\mathrm{NH}$, Unnikrishnan $\mathrm{S}$, Ramachandra JA, et al. Evaluation of relative position of mandibular foramen in children as a reference for inferior alveolar nerve block using orthopantamograph. J Clin Diagn Res 2017;11(3):ZC71-ZC74. DOI: 10.7860/JCDR/2017/25453.9585.

14. Hwang TJ, Hsu SC, Huang QF, et al. Age changes in location of mandibular foramen. Zhonghua Ya Yi Xue Hui Za Zhi 1990;9:98-103.

15. Ashkenazi M, Taubman L, Gavish A. Age-associated changes of the mandibular foramen position in anteroposterior dimension and of the mandibular angle in dry human mandibles. Anatom Record 2011;294(8):1319-1325. DOI: 10.1002/ar.21429.

16. Do Nascimento Pereira P, Fernandes Â, Gugisch RC, et al. Radiographic assessment of the mandibular foramen in children: focus on anesthetic procedures. Archiv Oral Res 2013;9(3):279-283.

17. Gershenson A, Nathan $H$, Luchansky E. Mental foramen and mental nerve: changes with age. Acta Anat(Basel) 1986;126(1):21-28. 